Preprint

UCRL-JC-147952

\title{
Detection of Materials of Interest to Non Proliferation: A Novel Approach
}

F. Ze, B.R. Tittmann, P.M. Lenahan

This article was submitted to Unattended Radiation Sensor Systems for Remote Application(URSSRA) Workshop, Carnegie Institution, Washington, DC, April 15-17, 2002

U.S. Department of Energy

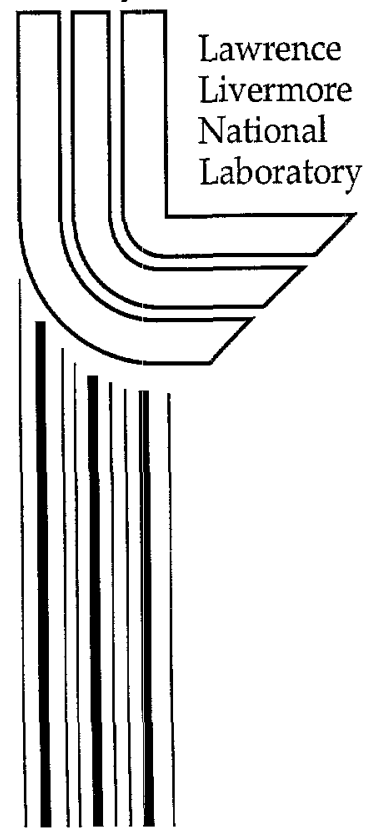

\section{April 11, 2002}




\section{DISCLAIMER}

This document was prepared as an account of work sponsored by an agency of the United States Government. Neither the United States Government nor the University of California nor any of their employees, makes any warranty, express or implied, or assumes any legal liability or responsibility for the accuracy, completeness, or usefulness of any information, apparatus, product, or process disclosed, or represents that its use would not infringe privately owned rights. Reference herein to any specific commercial product, process, or service by trade name, trademark, manufacturer, or otherwise, does not necessarily constitute or imply its endorsement, recommendation, or favoring by the United States Government or the University of California. The views and opinions of authors expressed herein do not necessarily state or reflect those of the United States Government or the University of California, and shall not be used for advertising or product endorsement purposes.

This is a preprint of a paper intended for publication in a journal or proceedings. Since changes may be made before publication, this preprint is made available with the understanding that it will not be cited or reproduced without the permission of the author.

This report has been reproduced directly from the best available copy.

Available electronically at http://www.doe.gov/bridge

Available for a processing fee to U.S. Department of Energy and its contractors in paper from

U.S. Department of Energy

Office of Scientific and Technical Information

P.O. Box 62

Oak Ridge, TN 37831-0062

Telephone: (865) 576-8401

Facsimile: (865) 576-5728

E-mail: reports@adonis.osti.gov

Available for the sale to the public from

U.S. Department of Commerce

National Technical Information Service 5285 Port Royal Road Springfield, VA 22161

Telephone: (800) 553-6847

Facsimile: (703) 605-6900

E-mail: orders@ntis.fedworld.gov

Online ordering: http://www.ntis.gov/ordering.htm

OR

Lawrence Livermore National Laboratory Technical Information Department's Digital Library http://www.llnl.gov/tid/Library.html 


\title{
Detection of Materials of Interest to Non Proliferation: A Novel Approach.
}

\author{
Frederic Ze* Bernhard R. Tittmann ${ }^{\dagger}$, and P. M. Lenahan ${ }^{\dagger}$ \\ * Lawrence Livermore National Lab, Livermore, California. \\ ${ }^{\dagger}$ The Department of Engineering Science and Mechanics, \\ Pennsylvania State University, University Park, Pennsylvania.
}

\begin{abstract}
We propose the development of a novel detector that can locate and identify materials of interest to Nuclear Arms Non Proliferation. The device will combine nuclear acoustic resonance (NAR) with superconducting quantum interference device (SQUD) widely used in nuclear magnetic resonance (NMR), geophysics, nondestructive evaluations, and biomagnetism, to name only few. NAR works like NMR. Thus resonant absorption (of applied ultrasonic energy) by a nuclear spin system occurs when the ultrasonic frequency is equal to the appropriate frequency separations between the magnetic nuclear energy levels. Ultrasonic energy couples to the nuclear spin system via spin-phonon interaction. The resulting nuclear acoustic resonance can be detected via the changes in (a) ultrasonic attenuation, (b) ultrasonic velocity, (c) material magnetization, (d) or nuclear magnetic susceptibility, all of which carries "intrinsic and unique signatures" of the material under investigation. The device's sensitivity and penetration depth (into metals) will be enhanced by incorporating SQUID technology into the design. We will present the details of interaction physics and outline a plan of action needed to successfully transform the concepts into a practical detector.
\end{abstract}

Work performed under the auspices of the U.S. Department of Energy by the University of California, Lawrence Livermore National Laboratory under Contract No. W-7405-Eng-48.

\section{INTRODUCTION}

We propose the development of a novel device to detect and identify smuggled materials that might be used in a weapons program or for terrorist activities. Its design will be based on Nuclear Acoustic Resonance (NAR) principles, and will incorporates SQUID (superconducting quantum interference device) to facilitate detection through metal barriers. The potential advantages of the device are as follows:

- The device will work where nuclear radiation detection is not possible.

- The device will be applicable to all materials of interest to Non Proliferation. 
- The incorporation of SQUID technology into the design will greatly enhance the device's sensitivity.

- The incorporation of SQUID technology will also help detect those metallic spin systems that are hard to access using room temperature nuclear acoustic resonance.

- A unique advantage of the concept is that the technique will not rely on nuclear radiation detection. The device's design principle and main objective will be to detect and identify fissile material even if steps had been taken by would-be smugglers to completely shield the material as a radiation source. Thus a successful development of the device could have enormous potential for the detection and identification of concealed fissile material, a key and still unresolved issue facing Non Proliferation and counterterrorism. Other potential advantages of the technique nclude the following:

- It will be highly sensitive: the technology upon which it will be built has been shown to detect extremely minute changes induced in the material's properties by an applied field in a wide range of scientific disciplines such as acoustics, nuclear magnetic resonance, geophysics and biology.

- The technique is highly penetrating. It has the potential to probe things deeply buried into solid or liquid material structures, including metals or alloys, a key attribute desired for detecting and identifying objects hidden inside thick metal boxes.

- The incorporation of the SQUTD technique makes it possible to detect those metallic spin systems that would be otherwise hard to access with room temperature NAR, like (a) nuclei with negligibly small gyromagnetic ratios; (b) metals, alloys, and metal hydrides in which spectral lines are broadened by quadrupolar or demagnetization effects; (c) and spin-lattice systems displaying long relaxation times because the maximum signal to noise ratio with SQUID is independent of relaxation times.

The objectives of this article are:

(1) To give a brief summary of how nuclear acoustic resonance works

(2) To briefly describe the two particular NAR interactions which we will attempt to explore and exploit during a feasibility study

(3) To show the derivation of expressions for the measurable quantities from which unique signatures of materials can be inferred.

(4) And to quickly summarize the NAR SQUID detection technique. The actual design of a prototype device will be undertaken only after our feasibility studies have been completed, including computer simulations and preliminary laboratory confirmation of computer codes 


\section{Nuclear Acoustic Resonance Explained}

Much of the material used in this summary was drawn from the listed references, and no claim to the originality of the concepts and principles is herein made by the authors.

Although the first paper on NAR was published as far back as $1956,{ }^{(1)}$ the theory of nuclear acoustic resonance is still relatively new to most physicists and engineers. Because it became increasingly clear that NAR can probe deep into conductors where magnetic resonance is ineffective, more and more material scientists started to seriously experiment with nuclear acoustic resonance for material characterization. ${ }^{(2-}$ 10)

In summary nuclear acoustic resonance works like nuclear magnetic resonance (NMR):

1. It induces shifts in the target material's nuclear spin energy levels in the presence of an externally applied constant magnetic field.

2. The energy levels affected are the same for NAR and NMR.

3. Energy shifts are not equal, but are closely related.

Basically, when a radio frequency (RF) acoustic wave is launched into a material object it couples to the material lattice where phonons of the same frequency as the acoustic wave are created. Phonons in turn perturb both the inter-nuclear distances and the overall charge distribution around nuclear sites. Charge perturbation creates electric field gradients which nuclear spins interact with via nuclear magnetic moments and nuclear electric quadrupoles, leading to the splitting of spin energy levels in the presence of a static magnetic field similarly to the nuclear magnetic resonance.

\section{Two appealing NAR coupling mechanisms}

Acoustic energy can couple to the material's nuclear spin system via various electric and magnetic interactions: ${ }^{(10)}$

1. The dynamic electric quadrupole interaction in which the acoustic wave dynamically couples to the electric quadrupole moments of the nuclear pins.

2. The magnetic dipole (or Alpher-Rubin) interaction ${ }^{(6)}$ in which the acoustic wave couples to the magnetic moments of the nuclear spins

3. The hexadecapole interaction which is via acoustic wave coupling to the electric hexadecapole (16-pole) moments of the nuclear spins. 
4. Or magnetic dipole-dipole interaction, in which acoustic wave couples to the material via magnetic interactions among nuclear spin themselves.

Since these various mechanisms couple to different multipole moments of the nuclear spins, each one leads to a different set of values for the observed interaction intensities, angular dependence, line widths and line shapes, given the same experimental conditions.

In terms of signal intensities, empirically the most important nuclear spin-phonon interactions in NAR are (1) the electric quadrupole and (2) the magnetic dipole interactions (or Alpher-Rubin coupling). These are the two mechanisms we want to explore during feasibility staudies.

1 The electric quadrupole interaction is the most effective mechanism for coupling acoustic waves in nonconductors (and some conductors) to nuclear systems with spin angular number $I>I / 2$

2 The Alpher-Rubin (magnetic dipole) interaction is effective only in metals. It depends on the electromagnetic interaction of conduction electrons with the acoustic wave in the presence of an externally applied static magnetic field. Furthermore, because the coupling is to the magnetic dipole moments of the nuclear spins, dipolar coupling is for all values of the nuclear spin quantum number, including $I=1 / 2$.

There are many other nuclear spin-phonon coupling mechanisms, but the above two are more appealing in terms of material characterization applications.

\section{Theoretical derivation for measurable quantities}

We are now show how the measurable variables are theoretically predicted. In practice NAR coupling is experimentally measured via two sets of variables. The coupling can be quantified by measuring NAR induced changed in acoustic wave attenuation and dispersion. Theoretically it will be seen that the measurement of those two quantities can immediately lead to the determination of NAR induced changed in material magnetic susceptibility. The other way of quantifying the coupling is by measuring NAR induced changes in material magnetization. In either case all these variables can be theoretically derived and numerically estimated using computer simulations. 


\section{Measurable 1. Acoustic Absorption Coefficient. ${ }^{(10)}$}

The acoustic power (per unit area) propagating in the material can be written as

$$
P_{o}=\varepsilon \mathrm{V}
$$

where $\varepsilon$ is the energy density of the acoustic wave in the material, and $\mathbf{v}$ is the sound speed. The coupling of the acoustic wave to the material lattice creates $\boldsymbol{n}_{p}$ phonons of the same frequency as the sonic wave. Thus the energy in the material is

$$
\varepsilon=\frac{n_{p} h v}{V}
$$

where $V$ is the sample's volume. The acoustic power density per unit area becomes:

$$
P_{o}=\frac{n_{p} h v}{V} \mathrm{~V}
$$

Under resonant conditions, a fraction of this power couples to the material's nuclear spin system, causing a transition between spin states $\boldsymbol{m}$ and $\boldsymbol{m}$ '. This corresponds to

$$
P_{n}=\Delta n h W_{m, m} .
$$

where $h v=E_{m}-E_{m^{\prime}}$ is the energy level shift between the two spin states as the result of energy exchanged between the acoustic wave and the spin system via the material lattice, $\Delta n=n_{m}-n_{m^{\prime}}$ is the equilibrium population difference per unit volume between $\mathrm{m}$ and $\boldsymbol{m}$ ', and $\boldsymbol{W}_{\boldsymbol{m}, \boldsymbol{m}}$, is the probability per unit time that a spin makes a transition from an initial state $\boldsymbol{m}$ to a final state $\boldsymbol{m}$.

Unless the acoustic wave energy absorption reaches a saturation level, a Boltzmann distribution of spins can be assumed, and in the $h v \ll \kappa_{\mathrm{B}} T$ limit, we can write the population difference as

$$
\Delta n=n_{m}-n_{m^{\prime}}=\left[\frac{N e^{-E_{m} / \kappa_{B} T}}{\sum_{m^{\prime}=-I}^{I} e^{-E_{m^{\prime}} / \kappa_{B} T}}\right]\left[e^{h \nu / \kappa_{B} T}-1\right] \approx \frac{N}{(2 I+1)} \frac{h v}{\kappa_{B} T}
$$

Acoustic power density can then be written as 


$$
P_{n}=\frac{N}{(2 I+1)} \frac{(h v)^{2}}{\kappa_{B} T} \sum_{m} W_{m, m^{\prime}}
$$

Defining the acoustic power absorption coefficient via $2 \alpha_{n}=\frac{P_{n}}{P_{o}}$, we then have:

$$
\alpha_{n}=\left(\frac{N}{2 I+1}\right)\left(\frac{h v}{\kappa_{B} T}\right) \frac{V}{2 n_{p} \mathrm{v}} \sum_{m} W_{m, m^{\prime}}
$$

The probability per unit time is found via Fermi Golden Rule, that is,

$$
W_{m, m^{\prime}}=\frac{1}{4 \hbar^{2}}\left|\left\langle p^{\prime}, m^{\prime}\left|H_{s-p}\right| p, m\right\rangle\right|^{2} g(v)
$$

where $p$ and $p^{\prime}=$ the initial and the final phonon states, respectively;

$\boldsymbol{H}_{s-p}=$ the spin-phonon interaction's Hamiltonian;

$g(v)=$ the spin absorption line shape function such that it peaks near the resonance frequency $\boldsymbol{v}_{\text {res }}$, and obeys the normalization requirement

$$
\int_{0}^{\infty} g(v) d v=1
$$

After substitution, the acoustic absorption coefficient is written as

$$
\alpha_{n}=\left(\frac{N}{2 I+1}\right)\left(\frac{\pi v}{\kappa_{B} T}\right)\left(\frac{V}{4 \hbar n_{p} \mathrm{v}}\right) g(v) \sum_{m}\left|\left\langle p^{\prime}, m^{\prime}\left|H_{s-p}\right| p, m\right\rangle\right|^{2}
$$

To calculate the matrix elements $\left|\left\langle p^{\prime}, m^{\prime}\left|H_{s-p}\right| p, m\right\rangle\right|$ of the nuclear spin-phonon interaction, we must first know (1) the energy levels of the nuclear spin system of the material under investigation; (2) and the physical mechanism responsible for the coupling of the acoustic wave to the nuclear spin of that material.

Energy levels can be determined from the knowledge of the nuclear properties of the material such as 
- the spin quantum number $I$

- the nuclear magnetic dipole moment

- the nuclear electric quadrupole moment

- the experimentally determined static quadrupole interaction

- and the magnitude and direction of the externally applied static magnetic field.

The second requirement involves explicit expressions for the dynamic interactions associated with NAR as will be illustrated later.

\section{Measurable 2. NAR Spin Susceptibility.}

\section{- Electric impedance analogy:}

Assuming the piezoelectric transducer is driven by a reasonably moderate voltage or current, a linear response by the material lattice to the acoustic excitation may be assumed in analogy to NMR, and the experimental set up can be described in terms of an electric impedance $Z(\omega)$ which changes due to nuclear spin transitions.

In a CW NAR experiment in which the driving acoustic frequency $\omega$ is kept at a fixed value corresponding to a particular resonance frequency $\omega_{n}$, a change of $\omega_{n}$ or of the phonon loss rate $1 / \tau$ causes a change in acoustic impedance for a particular acoustic component such that:

$$
\frac{\Delta_{j} Z}{Z_{o}}=\eta_{a} \frac{Q_{a}^{(n)}}{\omega_{n}}\left[\Delta_{j}(1 / \tau)-2 i \Delta_{j} \omega_{n}\right]
$$

where $Q_{a}^{(n)}=$ the acoustic quality factor at the mechanical resonance frequency $\boldsymbol{q}$, and $\Delta_{j}$ indicates a change due the $\boldsymbol{j} t \boldsymbol{h}$ mechanism for absorption or dispersion.

$$
\begin{aligned}
Z_{o} & =\text { the real part of } Z(\omega) \text { in the absence of spin transition. } \\
\mathrm{i} & =\sqrt{-1} \\
\eta_{a} & =\text { the acoustic filling factor }(\sim 1.0)
\end{aligned}
$$

Now in general the relative shift in frequency is numerically equal to the relative change in phase velocity, that is

$$
\Delta \omega_{n} / \omega_{n}=\Delta \mathrm{v}_{\mathrm{a}} / \mathrm{v}_{\mathrm{a}} \quad \text { and } \quad \Delta(1 / \tau)=2 \mathrm{v}_{\mathrm{a}} \Delta \alpha
$$

where $\mathrm{v}_{\mathrm{a}}$ is the phase velocity and $\Delta \alpha$ is the acoustic attenuation. 
In NMR, $\Delta Z / Z_{0}$ a good measure of detectability of nuclear spin transitions. By analogy we now define a NAR induced magnetic susceptibility via the NAR detectability relation:

$$
\left.\frac{\Delta_{j} Z}{Z_{o}}=i \eta_{a} Q_{a}^{(n)} \chi_{N A R j}(\omega)\right]
$$

where $\chi_{\text {NAR }}=$ the NAR induced magnetic susceptibility.

\section{- Derivation of a generalized expression for NAR induced magnetic susceptibility:}

The original scheme is due to $\mathrm{Kubo}^{(2)}$ and involves correlating the external acoustic perturbation Hamiltonian with experimental parameters.

The updated premise is as follows: ${ }^{(10)}$ A generalized linear response of a density operator $\rho(t)$ to an external force $f(t)$ can be obtained by applying the quantum mechanical version of Liouville's theorem on the density operator:

$$
i \hbar \frac{\partial \rho}{\partial t}=\left[H_{o}+H^{\prime}(t), \rho(t)\right]
$$

where $H_{o}$ is the Hamiltonian of the unperturbed physical system. The Fourier transform of this equation is (keeping only the first order term):

$$
\begin{aligned}
& \rho(\omega)=\frac{1}{i \hbar}\left[\tilde{H}^{\prime}(\omega), \rho_{o}\right] \\
& \text { where } \quad \tilde{H}^{\prime}(\omega)=\int_{0}^{\infty} e^{-i \omega t} e^{-(i / \hbar) H_{o} t} H^{\prime}(\omega) e^{(i / \hbar) H_{o} t} d t \\
& \text { and } \quad \rho_{o}=\text { the statistical operator at } t \rightarrow-\infty
\end{aligned}
$$

The above inverse Fourier transform of the perturbation Hamiltonian must be compatible with the assumption of a linear response of $\rho(t)$ to the driving acoustic time-dependent force $f(t)$. That is, the inverse transform must be of the form:

$$
\tilde{H}^{\prime}(\omega)=W(\omega) f(\omega)
$$

where $W(\omega)$ is the Fourier transform of the response operator $W(\tau)$; and $W(\tau)=0$ for $t<0$ and is independent of $f(\tau)$. 
A generalized susceptibility can now be written as

$$
\chi_{H^{\prime}}(\omega)=\frac{\operatorname{Tr}\left\{\rho(\omega) H^{\prime}(\omega)\right\}}{f(\omega) f(\omega)}
$$

Kubo originally conceived this idea for the NMR analysis. In the case of NAR where $f(t)$ is in the form of a strain sensor $\varepsilon$, it can be shown that the denominator takes two forms, namely

$$
[f(\omega)]^{2}=\rho_{s} v_{\mathrm{a}}^{2} \int_{\left(V_{s}\right)} d^{3} r\left[\varepsilon^{\prime}(r, \omega)\right]^{2} \text { and }[f(\omega)]^{2}=-\rho_{s} \int_{\left(V_{s}\right)} d^{3} r\left[\mathrm{v}_{i}(r, \omega)\right]^{2}
$$

Thus by substitution we can write the two cases for NAR induced material susceptibilities:

(a) $\quad \chi_{N A R}(\omega)=\frac{1}{i \hbar} \frac{\operatorname{Tr}\left\{\left[\tilde{H}^{\prime}(\omega), \rho_{o}\right] H^{\prime}(\omega)\right\}}{\rho_{s} \int_{\left(V_{s}\right)} d^{3} r\left[\mathrm{v}_{i}(r, \omega)\right]^{2}}$

(b) and $\chi_{N A R}(\omega)=-\frac{1}{i \hbar} \frac{\operatorname{Tr}\left\{\left[\tilde{H}^{\prime}(\omega), \rho_{o}\right] H^{\prime}(\omega)\right\}}{\rho_{s} \mathrm{v}_{\mathrm{a}}^{2} \int_{\left(V_{s}\right)} d^{3} r\left[\epsilon^{\prime}(r, \omega)\right]^{2}}$

\section{- Case for an Acoustic Wave in a Metal:}

The appropriate spin-phonon Hamiltonian of an acoustic wave in a metal can be written as

$$
H^{\prime}(t)=H_{D}^{\prime}(t)+H_{Q}^{\prime}(t)
$$

where

$$
H_{D}^{\prime}(t)=-\int d^{3} r m(r) b(r, t)
$$

and

$$
H_{Q}(t)=-\int d^{3} r Q(r): \nabla V(r, t)
$$

In these expressions, $H_{D}^{\prime}(t)$ is the dynamic Alpher-Ruber term, and represents the coupling of nuclear spins to the acoustically induced magnetic field; $H_{Q}(t)$ is the dynamic electric quadrupole term which represents the coupling of nuclear spins to the acoustically induced electric field gradient; $\mathbf{m}(\mathbf{r})=$ nuclear spin magnetization vector; $\nabla V(r, t)$ is nuclear 
spin magnetization vector; and $\mathbf{Q}(\mathbf{r})$ is the tensor operator of the nuclear electric quadrupole moment density.

So that we now can write

$$
\left.\operatorname{Tr}\left\{\left[\tilde{H}^{\prime}(\omega), \rho_{o}\right] H^{\prime}(\omega)\right\}=\sum_{i, j=D \text { or } Q} \operatorname{Tr}\left\{\tilde{H}_{i}^{\prime}(\omega), \rho_{o}\right] H_{j}^{\prime}(\omega)\right\}
$$

After some algebra and substitution into equations 21 and 22, we obtain $\boldsymbol{a}$ generalized expression for the NAR induced susceptibility in conductors

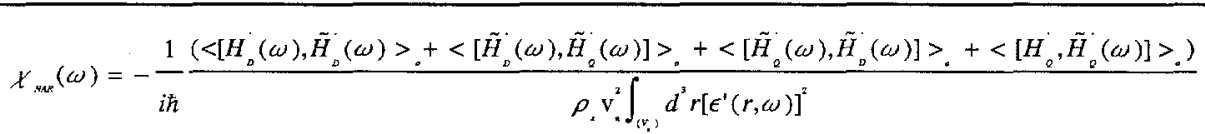

Thus, there are Three Contributions to the NAR-induced Susceptibility in a Conductor:

(1) A contribution from NAR Dipole Coupling induced (or pure AlpherRubin) Susceptibility given by

$$
\chi_{N A R_{(D \text { or A-R }}}(\omega)=-\frac{1}{i \hbar} \frac{<\left[H_{D}^{\prime}(\omega), \tilde{H}_{D}^{\prime}(\omega)\right]>}{\rho_{s} \mathrm{v}_{\mathrm{a}}^{2} \int_{\left(V_{s}\right)} d^{3} r\left[\epsilon^{\prime}(r, \omega)\right]}
$$

(2) A contribution from a "Pure dynamic Quadrupole" Coupling induced Susceptibility given by

$$
\chi_{N A R_{(Q)}}(\omega)=-\frac{1}{i \hbar} \frac{<\left[H_{Q}^{\prime}(\omega), \tilde{H}_{Q}^{\prime}(\omega)\right]>_{o}}{\rho_{s} \mathrm{v}_{\mathrm{a}}^{2} \int_{\left(V_{s}\right)} d^{3} r\left[\varepsilon^{\prime}(r, \omega)\right]^{2}}
$$

(3) And a contribution is due to an "Interference Susceptibility" between the dynamic Alpher-Rubin and Quadrupole, and is given by

$$
\chi_{N A R_{\text {(nit) }}}(\omega)=-\frac{1}{i \hbar} \frac{<\left[H_{D}^{\prime}(\omega), \tilde{H}_{Q}^{\prime}(\omega)\right]+\left[H_{Q}^{\prime}(\omega), \tilde{H}_{D}^{\prime}(\omega)\right]>_{o}}{\rho_{s} \mathrm{v}_{\mathrm{a}}^{2} \int_{\left(V_{s}\right)} d^{3} r\left[\varepsilon^{\prime}(r, \omega)\right]^{2}}
$$


Example 3. Explicit NAR Quadrupole Spin Susceptibility in a Metal. ${ }^{(10)}$

The Hamiltonian for a NAR quadrupole interaction is given by

$$
H_{Q}=\frac{e^{2} q Q}{4 I(2 I-1)}\left\{3 I_{z^{\prime}}^{2}-I(I+1)+\frac{1}{2} \eta\left(I_{+}^{2}+I_{-}^{2}\right)\right\}
$$

where $\quad I_{ \pm}=\left(I_{x} \pm i I_{y}\right) ; \quad$ and $\quad \eta=\frac{\partial^{2} V / \partial x \partial x-\partial^{2} V / \partial y \partial y}{\partial^{2} V / \partial z \partial z}$

and

$$
e q=\partial E_{z} / \partial z=\sum_{j e_{j}}\left(3 \cos ^{2} \theta_{j}-1\right) r_{j}^{-3}
$$

the magnitude of the axially symmetric field gradient due to all the charges $\mathbf{e}_{\mathrm{j}}$ external to the nucleus, $r_{j}$ and $\theta_{j}$ are the coordinates (with origin at nuclear center) of the element charge $\mathbf{e}_{\mathbf{j}}$.

When this expression for the quadrupole is used in the matrix element for NAR induced transition probability calculations, It is seen that ,both $\Delta \boldsymbol{m} \pm 1$ and $\Delta \boldsymbol{m}= \pm 2$ transitions are allowed in quadrupole interactions. And so in this case the NAR induced $\chi_{N A R_{i O}(2)}$ is given by

$$
\begin{aligned}
& \chi_{\operatorname{NAR}_{(Q)}}=\chi_{N A R 1}+\chi_{N A R 2} \\
& \text { where } \chi_{\text {NARI }}(\omega)=D_{1}(\omega) \frac{\int_{\left(V_{r},\right.} d^{3} r V_{+1}(r, \omega) V_{-1}(r, \omega)}{\int_{\left(\mathbb{V}_{r}\right)} d^{3} r\left[\varepsilon^{\prime}(r, \omega)\right]^{2}} \quad \text { (for } \Delta m= \pm 1 \text { transitions) } \\
& \chi_{M A R 2}(\omega)=D_{2}(\omega) \frac{\int_{\left(V_{1}\right)} d^{3} r V_{+2}(r, \omega) V_{-2}(r, \omega)}{\int_{\left(V_{2}\right)} d^{3} r^{\prime}\left[\varepsilon^{\prime}(r, \omega)\right]^{2}} \quad \text { (for } \Delta m= \pm 2 \text { transitions) } \\
& D_{1}(\omega)=\frac{\pi A^{2}}{16 \hbar \rho_{s} v_{\mathrm{a}}^{2}} \sum_{m=-1}^{I-1} \frac{N_{m}-N_{m+1}}{V_{s}}(2 m+1)^{2} \times f_{I}^{2}(m)\left[g\left(\omega-\omega_{m+1, m}\right)-g\left(\omega+\omega_{m+1, m}\right)\right]
\end{aligned}
$$

\section{Measurable4. NAR Energy Levels Shifts in Quadrupole Interactions.}

The NAR induced quadrupole-split energy levels are calculated using the above Hamiltonian for phonon-spin quadrupolar interactions: 


$\begin{aligned} E & =E_{m}+\left(m\left|H_{q}\right| m\right) \\ & =E_{m}+\left(m\left|H_{Q}=\frac{e^{2} q Q}{4 I(2 I-1)}\left\{3 I_{z^{\prime}}^{2}-I(I+1)+\frac{1}{2} \eta\left(I_{+}^{2}+I_{-}^{2}\right)\right\}\right| m\right) \\ & =-m \hbar \omega_{o}+\hbar \omega_{q}\left[m^{2}-\frac{1}{3} I(I+1)\right] \\ \text { where, } \quad \omega_{q} & =\frac{e^{2} q Q}{\hbar 2 I(2 I-1)} \frac{3}{4}\left(3 \cos ^{2} \theta-1\right) \\ \theta= & \text { the angle between the static field } H_{\circ} \text { and the axis of symmetry of q }\end{aligned}$

Thus NAR nuclear spin energy levels shifts are relatively easy to calculate numerically, given the material properties, the transition rules as seen above, the experimental configuration, and the spin-phonon interaction of interest.

\section{Measurable 5. Transition Probabilities for NAR Quadrupole Interactions $^{(10)}$.}

Transition probabilities are similarly calculated using the same quadrupole interaction Hamiltonian given by equation (31):

$$
W_{m, m^{\prime}}=\frac{1}{4 \hbar^{2}}\left|\left\langle p^{\prime}, m^{\prime}\left|H_{Q}\right| p, m\right\rangle\right|^{2} g(v)
$$

Thus we have

$$
\begin{aligned}
W_{Q_{1}} & =W_{m, m+1}=\frac{1}{4 \hbar^{2}}\left|\left\langle p+1, m+1\left|H_{Q_{1}}\right| p, m\right\rangle\right|^{2} g(v) \\
& =6\left(\frac{A}{4 \hbar}\right)^{2} \xi_{ \pm}^{2} g_{Q_{1}}(v)\left|V^{ \pm 1}\right|^{2}
\end{aligned}
$$

and

$$
\begin{aligned}
W_{Q_{2}} & =W_{m, m+2}=\frac{1}{4 \hbar^{2}}\left|\left(p+2, m+2\left|H_{Q_{2}}\right| p, m\right)\right|^{2} g(v) \\
& =6\left(\frac{A}{4 \hbar}\right)^{2} \eta_{ \pm}^{2} g_{Q_{2}}(v)\left|V^{ \pm 2}\right|^{2}
\end{aligned}
$$

where: 


$$
\begin{aligned}
& \xi_{ \pm}=(2 m \pm 1)[(I \pm m+1)(I \mp m)]^{\frac{1}{2}} ; \eta_{ \pm}=[(I \mp m)(I \mp m-1)(I \pm m+1)(I \pm m+2)]^{\frac{1}{2}} \\
& V^{ \pm 1}= \pm \frac{1}{4}\left(6^{\frac{1}{2}}\right) e q \sin \theta \cos \theta e^{ \pm i \phi} \\
& V^{ \pm 2}=\frac{1}{8}\left(6^{\frac{1}{2}}\right) e q \sin ^{2} \theta e^{ \pm 2 i \phi}
\end{aligned}
$$

$\phi$ is the angle between the projection of symmetry axis on the xy-plane and the Z-axis; $g_{Q_{1}(v) \text { and }} g_{Q_{2}(v)}$ are the shape factors for the $\Delta m= \pm l$ and $\Delta m=$ \pm 2 transitions, respectively. Other variables have been defined previously.

\section{Specific Case for material with spin quantum number $\underline{I=7 / 2}$}

The effects of NMR and NAR on the Zeeman energy shifts for materials

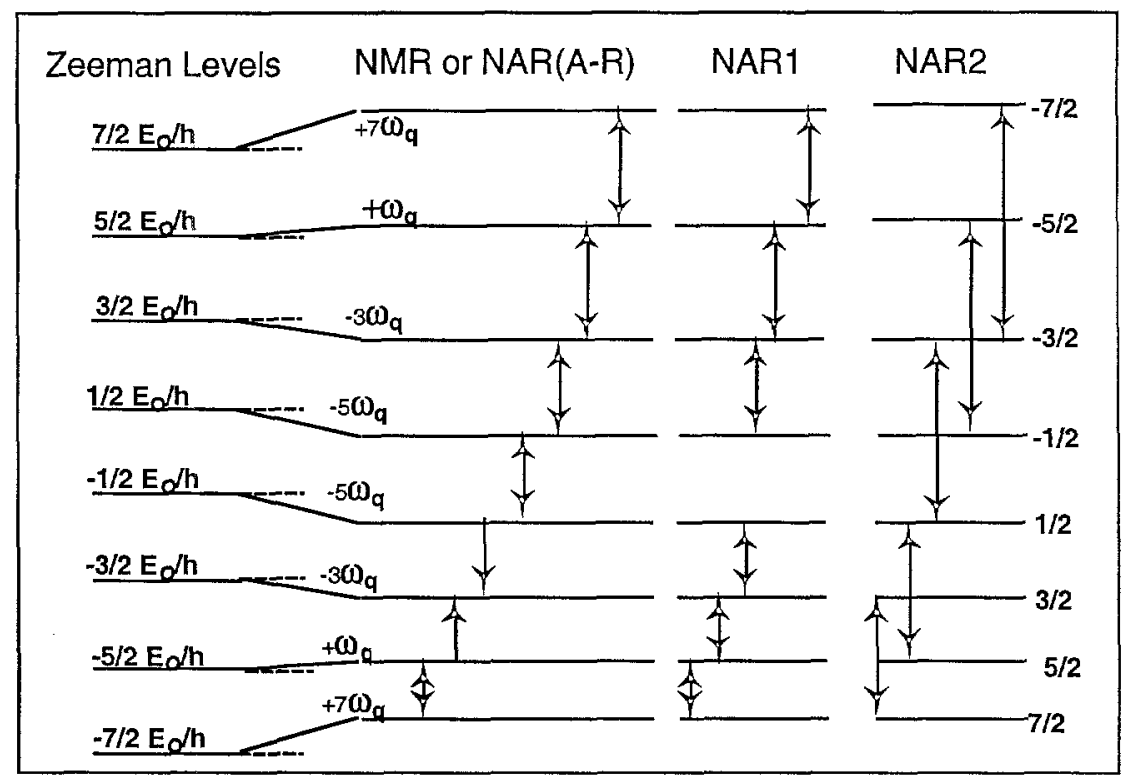

Figure 1 Quadrupole-shifted energy levels and resulting spectra for $I=7 / 2$ with spin quantum number $\mathrm{I}=7 / 2$ are shown in Figure 1. Arrows show allowed transitions for each case. The figure shows allowed transitions for the two quadrupolar interactions NAR1 $(\Delta \mathrm{m}= \pm 1)$ and NAR2 $(\Delta \mathrm{m}= \pm 2)$. Neither case allows a $\Delta \mathrm{m}= \pm 1 / 2$ transition. In the same figure we also show the transitions due to nuclear magnetic resonance (NMR) as well as those due to the special case of NAR (shown as NAR(A-R) involving the 
interaction of nuclear magnetic moments with the acoustically induced electric field gradients. That is, the Alpher-Rubin coupling (valid only in metals). The figure shows that unlike the quadrupolar couplings (NAR1 and NAR2), the NAR(A-R) energy shifts and allowed transitions are exactly the same as those of NMR. This comparison serves to illustrate the fact NMR and NAR affect the same nuclear spin energy levels, but the resulting transitions and probabilities and not necessarily the same in the case of nuclear electric quadrupole interactions.

The relative signal levels can also be predicted using the acoustic absorption formula developed earlier (equation 10), by substituting the quadrupole interaction Hamiltonian expression. The resulting relations are:

$$
\begin{aligned}
& \alpha_{Q_{1}}=\frac{3 \pi^{2}}{8} \frac{N e^{2} Q^{2}}{\rho v^{3} \kappa_{B} T} v^{2} g_{Q_{1}}(v) f_{Q}(I) \frac{\left|V^{ \pm 1}\right|^{2}}{\varepsilon^{2}} \\
& \alpha_{Q_{2}}=\frac{3 \pi^{2}}{8} \frac{N e^{2} Q^{2}}{\rho v^{3} \kappa_{B} T} v^{2} g_{Q_{2}}(v) f_{Q}(I) \frac{\left|V^{ \pm 2}\right|^{2}}{\varepsilon^{2}} \\
& \alpha_{N A R(A-R)}=\frac{\pi^{2}}{3} \frac{N h^{2}}{\rho v^{3} \kappa_{B} T} v^{4} g_{D}(v) f_{N A R(N-A)}(I) \\
& \text { with } f_{Q}(I)=\frac{\sum_{m=-I}^{I} \xi^{2}(m)}{(2 I)^{2}(2 I-1)^{2}(2 I+1)} \text { and } f_{N A R(N-A)}(I)=I(I+1)
\end{aligned}
$$

The quantities $f_{Q}(I)$ and $f_{N A R(N-A)}$ predict the relative strengths of the Alpher-Rubin dipolar coupling and the quadrupolar coupling. In fact for a material with spin quantum number $\mathrm{I}=7 / 2$, the quadrupolar value $f_{Q}=1 / 4$, while the NAR(A-R) value is $f_{N A R(A-R)}=63 / 4$. This difference is quite important because in the SQUID-NAR technique, it is the Alpher-Rubin coupling which plays a role if the technique is used to study a conducting material. Another important factor in the Alpher-Rubin coupling is the intensity is proportional to $v^{4}$ as shown in equation 45 , which again add to the strength of signal intensity.

On the other hand, signal intensities in quadrupolar interactions are proportional to the square of the nuclear electric quadrupole moments as shown in equations, which contribute to their relative strength when for materials with relatively large quadrupole moments. 
Table 1 gives a summary of corresponding transition frequencies and their relative probabilities. Again nuclear magnetic resonance transition frequencies and relative probabilities are also shown in the table for comparison. In the table the frequency shifts are computed from the term

$$
\Delta=\frac{e^{2} q Q}{h} \frac{\left(3 \cos ^{2} \theta-1\right)}{8 I(2 I-1)}
$$

Table 1: Transition Frequencies and Probabilities in NAR for $I=7 / 2$.

\begin{tabular}{|cccccccc|}
\hline \multicolumn{9}{c}{ NMR } & \multicolumn{2}{c}{ NAR1 } & \multicolumn{3}{c|}{ NAR2 } \\
Trans & Freq. & Prob. & Freq. & Prob. & Trans & Freq. & Prob. \\
$+\frac{5}{2} \leftrightarrow+\frac{7}{2}$ & $\omega_{o}+18 \Delta$ & 7 & $\omega_{0}+18 \Delta$ & 21 & $+\frac{3}{2} \leftrightarrow+\frac{7}{2}$ & $2 \omega_{0}+30 \Delta$ & 7 \\
$+\frac{3}{2} \leftrightarrow+\frac{5}{2}$ & $\omega_{0}+12 \Delta$ & 12 & $\omega_{0}+12 \Delta$ & 16 & $+\frac{1}{2} \leftrightarrow+\frac{5}{2}$ & $2 \omega_{o}+18 \Delta$ & 15 \\
$+\frac{1}{2} \leftrightarrow+\frac{3}{2}$ & $\omega_{0}+6 \Delta$ & 15 & $\omega_{0}+6 \Delta$ & 5 & $-\frac{1}{2} \leftrightarrow+\frac{3}{2}$ & $2 \omega_{0}+6 \Delta$ & 20 \\
$-\frac{1}{2} \leftrightarrow+\frac{1}{2}$ & $\omega_{0}$ & 16 & & & & & \\
$-\frac{3}{2} \leftrightarrow-\frac{1}{2}$ & $\omega_{o}-6 \Delta$ & 15 & $\omega_{0}-6 \Delta$ & 5 & $-\frac{3}{2} \leftrightarrow+\frac{1}{2}$ & $2 \omega_{o}-6 \Delta$ & 20 \\
$-\frac{5}{2} \leftrightarrow-\frac{3}{2}$ & $\omega_{0}-12 \Delta$ & 12 & $\omega_{0}-12 \Delta$ & 16 & $-\frac{5}{2} \leftrightarrow-\frac{1}{2}$ & $2 \omega_{0}-18 \Delta$ & 15 \\
$-\frac{7}{2} \leftrightarrow-\frac{5}{2}$ & $\omega_{0}-18 \Delta$ & 7 & $\omega_{0}-18 \Delta$ & 21 & $-\frac{7}{2} \leftrightarrow-\frac{3}{2}$ & $2 \omega_{0}-30 \Delta$ & 7 \\
\hline
\end{tabular}

The examples discussed here have served to illustrate the cause and effects relationships between the acoustic wave and the experimentally observable quantities which carry the unique signatures of the material under investigation. The expose also serves to signify the need for good computer simulation effort to obtain numerical estimates of various variables and to establish a baseline for the proposed detector specifications.

\section{SQUID Detection of NAR induced magnetization change}

As stated in the introduction, a major feature in the proposed NARbased detector will be the use of the SQUID. The SQUID based NAR detection $^{(2,7-9)}$ relies not on the observation acoustic radiation absorption 
or dispersion, but on the induced changes in the component of material magnetization which is parallel to an applied static magnetic field.

SQUID technique will confer to the device the ability to detect acoustically induced magnetization changes even if the latter were extremely weak. The induced transitions among the nuclear magnetic or nuclear quadrupole energy levels are the same whether they are observed via acoustic absorption/dispersion, or via changes in the z-component $\boldsymbol{M}_{\boldsymbol{z}}$ of the induced magnetization $M$. Because the SQUID detected NAR technique relies on the detection of changes $M_{z}$, it is sensitive to the magnetic flux $\Phi$ itself rather than to the $d \Phi / d t$. The maximum magnetization available when $\boldsymbol{M}_{\boldsymbol{z}}$ is varied from its equilibrium value $\boldsymbol{M}_{\boldsymbol{o}}$ is given by

$$
M_{z}=M_{o}=\chi_{o} H_{o}
$$

where $\chi_{o}$ the static nuclear susceptibility, and $\boldsymbol{H}_{o}$ is the applied magnetic field. This relation immediately links $\chi_{o}$ to the measurable quantity $\boldsymbol{M}_{\boldsymbol{z}}$. The maximum change in the magnetic flux $\Phi$ through a loop of area $A$ is given by:

$$
\Phi_{\max }=\left(4 \pi \chi_{o} H_{o}\right)\left(A \eta t_{x}\right)
$$

where $\eta i$ s a filling factor $\sim 1 / 2$, and $t_{x}$ is the flux transfer factor.

Thus rough estimates of the expected flux change $\Phi / \Phi_{o}$ in several metals can be made at the radio frequency SQUID for acoustic coupling to the nuclear spins.

For illustration a schematic diagram of a SQUID-assisted acoustomagnetic spectrometer once used to study transient nuclear spin-phonon interactions in metal antimony ${ }^{(9)}$ is shown in Figure 2 (minus the superconducting magnet, the superconducting shields and the complex fluxtransformer circuit). The various components of the spectrometer are shown in the figure. Also not shown is the computer-assisted data reduction component of the setup. The design of the SQUID-coupled device we propose will be based on the principle illustrated in this figure. The actual design of a prototype device will follow our expected feasibility studies during which issues such as signal to noise ratios, spin-lattice relaxation times, the ambiance induced effects from electronic circuitry, etc., will be investigated in additions to signal levels issues, transducers design, transducers-specimen couplings, data acquisition and analysis automation, among others. More specifically, among the issues the feasibility 


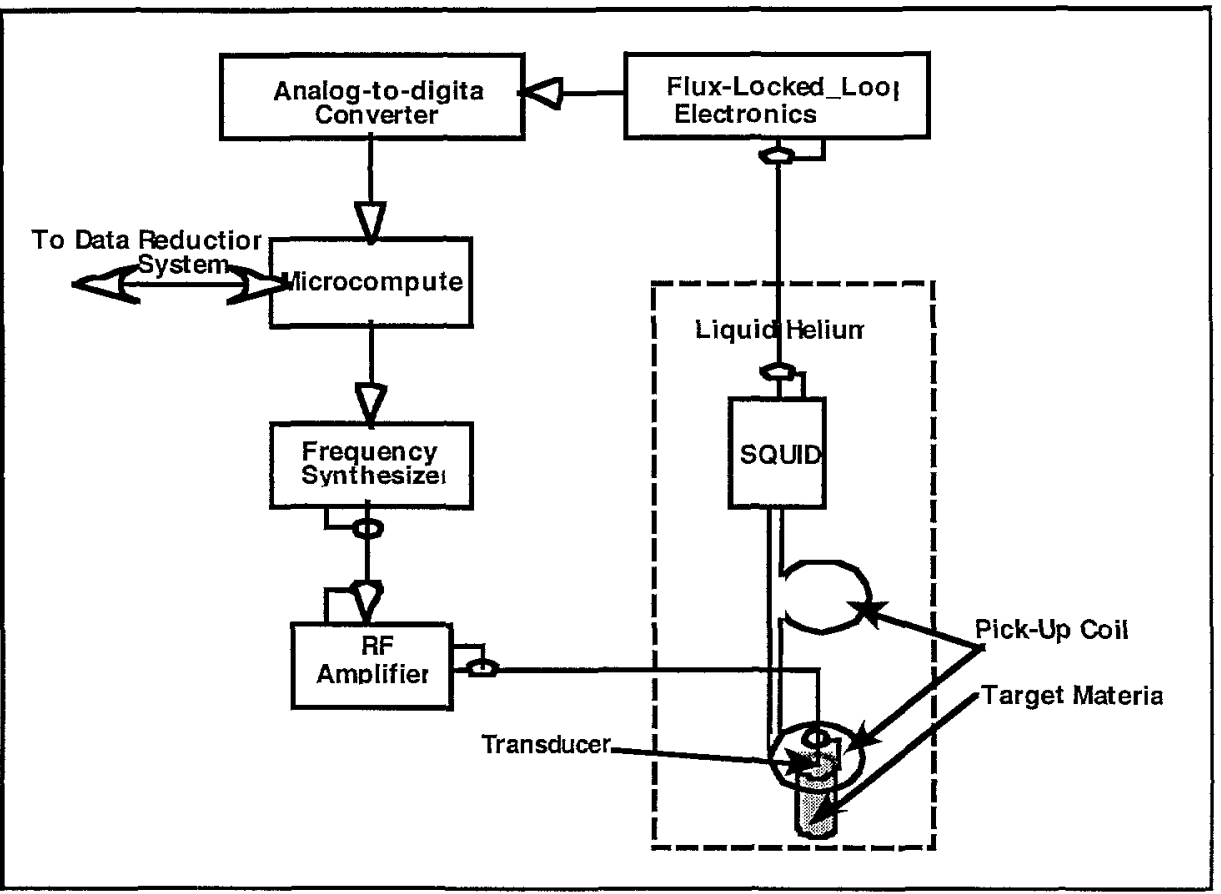

Figure 2. Schematic of a SDUID-acoustomagnetic spectrometer.

studies will address are, how signal intensities in terms of NAR-induced changes ultrasonic dispersion and attenuation, material magnetization, material susceptibility, signal to noise ratios, etcetera, scale relative to

- the operating temperature

- the object size and geometry

- the specimen housing box material's Z-number

- the specimen housing box material's thickness

- the ordering and variation of layers in the specimen housing box's thickness

- the housing box fill materials around the specimen to mimic intentional material camouflage used to elude detection

- transducer sizes and models, and transducer/objects interfacing technique (including air gaps).

The exercise will involve computer simulations and preliminary experimental validation of simulation results. It is hoped that the activites give us a much needed insight that will enable us to rationally narrow down the choices not only for later lab tests, but also for the final detector 
specifications needed in phase 2 of the project. Additionally we would also like to do similar tests using composite materials and materials with mixed isotopes (as opposed to pure elements). For the fact is that most practical situations would require the technique to work for such materials. . Most of the work for feasibility study will be performed at Penn State University in the Department or Engineering Science and Mechanics.

In summary, we want to do quality scientific work for a high risk scheme which, if successful, could meet a vital national security need.

\section{REFERENCES}

1. Proctor, W. G., and Robinson, W. A., Physical Review, 104, 5, 13441352 (1956).

2. Kubo, R. Tomita, and K., J. Phys. Soc. Jap., 9, 888-919 (1954).

3. Bolef, D. I., and Menes, M., Physical Review, 114, 6, 1441-1451 (1959).

4. Zimmerman, J. E., Cowen, J. A., and Silver, A. H., Applied Physics Letters, 9, 10 353-355 (1966).

5. Silver, A. H., and Zimmerman, J. E., Applied Physics Letters, 10, 5, 142145 (1967).

6. Fedders, P. A., Physical Review B, 1740, 1740-1743, (1973)

7. Webb, R. A., Rev. Sci. Instrum., 48, 12, 1585-1594 (1977).

8. Pickens, K. S., Mozurkwich, G., Bolef, D. I., and Sundfors, R. K., Physical Review Letters, 53, 2,156-159 (1984).

9. Pickens, K. S., Bolef, D. I., Holland, M. R., and Sundfors, R. K., Physical Review B, 30, 3644-3648 (1984).

10. Bolef, D. I., and Sundfors, R. K., Nuclear Acoustic Resonance, Harcourt Brace \& Company, Publishers, Boston, San Diego, New York, London, Syney, Tokyo, Toronto, 1993. 